\title{
Dynamical equations for time-ordered Green's functions: from the Keldysh time-loop contour to equilibrium at finite and zero temperature
}

\author{
H. Ness, L. K. Dash \\ Department of Physics, University of York, Heslington, York YO10 5DD, UK \\ European Theoretical Spectroscopy Facility (ETSF)
}

\begin{abstract}
We study the dynamical equation of the time-ordered Green's function at finite temperature. We show that the time-ordered Green's function obeys a conventional Dyson equation only at equilibrium and in the limit of zero-temperature. In all other cases, i.e. finite-temperature at equilibrium or non-equilibrium, the timeordered Green's function obeys instead a modified Dyson equation. The derivation of this result is obtained from the general formalism of the non-equilibrium Green's functions on the Keldysh time-loop contour. At equilibrium, our result is fully consistent with the Matsubara temperature Green's function formalism and also justifies rigorously the correction terms introduced in an ad hoc way with Hedin and Lundqvist. Our results show that one should use the appropriate dynamical equation for the time-ordered Green's function when working beyond the equilibrium zero-temperature limit.
\end{abstract}

PACS numbers: 71.10.Ca, 71.10.w, 71.15.m 


\section{Context}

Equilibrium, zero-temperature and finite-temperature Green's functions (GF) techniques based on many-body perturbation theory are widely used in electronic-structure and total-energy calculations. They are central for the calculation of the thermodynamical properties of many-body systems, as well as for the calculation of linear responses of systems under small time-dependent (or not) perturbations [1, 2, 3, 4, 5, 6, 7, 8].

Conventional equilibrium many-body GF approaches, at zero temperature, concentrate on the time-ordered GF and determine its dynamical equation (in the form of an equation of motion, or its integral form known as the Dyson equation) using a selfenergy which includes all the many-body interaction effects. The Matsubara formalism using time-ordered GF with imaginary time arguments (or imaginary Matsubara frequency in the dual Fourier representation) has been developed to deal with the finite temperature condition. Interestingly, not much has been done at finite-temperature for the time-ordered GF in real time or real frequency representation.

However, when one wants to study systems driven out of equilibrium by an external "force", as for example in the context of electron or heat current flow, or for any system driven by an external electromagnetic field (whether time-dependent or not), one needs to extend the equations for the dynamics of the quantum many-body interacting system to the non-equilibrium conditions.

For this, the non-equilibrium Green's function (NEGF) technique[2, 9, 10, 11, 12, 13, 14. has been widely used to calculate non-linear charge and heat transport properties of solids, mesoscopic and nanoscopic systems. Also known as the closed time-path formalism [15, 11], the NEGF formalism relies on an "artificial" time parameter that runs on a mathematically convenient time-loop contour (plus eventually an imaginary time for taking into account the initial correlation and statistical boundary conditions). It is a formal procedure that only has a direct physical meaning when one projects back the time parameters of the time-loop contour onto real times. It was introduced because it allows one to obtain self-consistent Dyson-like equations for the Keldysh Green's function using Schwinger's functional derivative technique. Transforming the Dyson equation to real time by varying the Keldysh time parameter over the time-loop contour results in a set of self-consistent equations for the different non-equilibrium Green's functions (advanced/retarded or lesser/greater). The NEGF technique is general and can treat non-equilibrium as well as equilibrium conditions, at zero and finite temperatures, within a single framework.

In this paper we focus on the dynamical equation of the time-ordered GF with real time argument, and we show that the time-ordered GF obeys a conventional Dyson equation only in a limiting case: at equilibrium and zero temperature. In any other cases, i.e. equilibrium at finite temperature or non-equilibrium, the time-ordered GF obeys instead a modified Dyson equation containing correction terms. These correction terms were introduced in an ad hoc way in the seminal work of Hedin and Lundqvist [16]. 


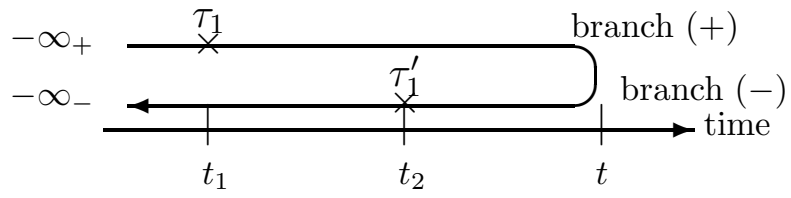

Figure 1. The time-loop Keldysh contour $C_{K}$ for an "artificial" time parameter ordering on a forward time evolution $(+)$ branch followed by a backward time evolution $(-)$ branch, the "turning" point $t$ is taken as $t \rightarrow \infty$.

We derive the modified Dyson equation for the time-ordered GF in a natural way from the Keldysh time-loop contour approach. We also show that for the equilibrium case at finite temperature, such correction terms can be obtained by a proper treatment of the imaginary time Matsubara finite-temperature Green's functions.

The paper is organized as follows: in section 2, we derive the modified Dyson equation for the time-ordered GF from the time-loop contour formalism. We then concentrate on the equilibrium condition. Section 3 shows explicitly the relation between Hedin and Lundqvist's seminal work on real-time GFs at finite temperature and our result. In section 4, we show that our result can also be obtained in by using Matsubara temperature GFs and a proper treatment of the analytical continuation rules. We conclude and discuss the importance of our result in section 5. Appendix A and Appendix B contain important and useful relations for the GFs and self-energies on the time-loop contour. In Appendix C we provide a concrete example of our general results for a specific system: the single site Hubbard model at finite temperature.

\section{Non-equilibrium Green's functions on the Keldysh contour $C_{K}$}

The Green's function on the Keldysh contour $C_{K}$ is defined as

$$
G(1,2)=-\mathrm{i}\left\langle\mathcal{T}_{C_{K}} \Psi(1) \Psi^{\dagger}(2)\right\rangle,
$$

where $(1,2)$ stands for a composite index for space $\mathbf{x}_{1,2}$ and time $\tau_{1,2}$ on the time-loop contour $C_{K}$. The time-loop contour $C_{K}$ consists of a chronological time evolution $(+)$ branch followed by an anti-chronological time evolution (-) branch [9, 10, 11, 13], as shown in Figure 1. The time ordering $\mathcal{T}_{C_{K}}$ of the product of fermion creation $\left(\Psi^{\dagger}\right)$ and annihilation $(\Psi)$ quantum fields is performed on the time-loop contour $C_{K}$.

The Green's function obeys the equation of motion on the contour $C_{K}$ [10, 13]:

$$
\left[i \partial_{\tau_{1}}-\bar{h}_{0}(1)\right] G\left(1,1^{\prime}\right)=\delta\left(1-1^{\prime}\right)+\int \mathrm{d} 3 \Sigma(1,3) G\left(3,1^{\prime}\right)
$$

and the adjoint equation reads

$$
\left[-i \partial_{\tau_{1}^{\prime}}-\bar{h}_{0}\left(1^{\prime}\right)\right] G\left(1,1^{\prime}\right)=\delta\left(1-1^{\prime}\right)+\int \mathrm{d} 2 G(1,2) \Sigma\left(2,1^{\prime}\right),
$$

where $\bar{h}_{0}(1)$ is the non-interacting Hamiltonian. 
Hence, in a integral form, $G\left(1,1^{\prime}\right)$ follows a Dyson-like equation

$$
G(1,2)=g(1,2)+\int \mathrm{d} 3 \mathrm{~d} 4 g(1,3) \Sigma(3,4) G(4,2),
$$

where $g(1,2)$ is the Green's function for the non-interacting system, i.e. $\left[i \partial_{\tau_{1}}-\right.$ $\left.\bar{h}_{0}(1)\right] g(1,2)=\delta(1-2)$, and the time integrations run on $C_{K}$. Eq. (4) is valid provided that, in the remote past, the system is unperturbed and non-interacting and that the adiabatic assumption for switching on the interaction is fulfilled, as usually assumed within the Keldysh formalism [9]. Furthermore the non-interacting Green's function $g(1,2)$ satisfies the Kubo-Martin-Schwinger boundary conditions (for example see Eq. (3.16) in [13]).

Upon the position of the time arguments $\left(\tau_{1}, \tau_{2}\right)$ on $C_{K}$, one obtains four different projections onto the real (normal) time arguments $\left(t_{1}, t_{2}\right)$, i.e. the four "Keldysh components" of $G\left(1,1^{\prime}\right): G^{\eta_{1} \eta_{2}}\left(\mathbf{x}_{1} t_{1}, \mathbf{x}_{2} t_{2}\right)$ with $t_{i}$ on the $\eta_{i}= \pm$ branch of $C_{K}$.

So $G^{++}\left(t_{1}, t_{2}\right)=-\mathrm{i}\left\langle\mathcal{T} \Psi(1) \Psi^{\dagger}(2)\right\rangle$ is the conventional time-ordered GF (though in the non-equilibrium conditions), and $G^{-+}\left(t_{1}, t_{2}\right)=-\mathrm{i}\left\langle\Psi(1) \Psi^{\dagger}(2)\right\rangle=G^{>}(1,2)$ and $G^{+-}\left(t_{1}, t_{2}\right)=\mathrm{i}\left\langle\Psi^{\dagger}(2) \Psi(1)\right\rangle=G^{<}(1,2)$ are automatically ordered knowing that any time on the branch (-) is later than any time on the branch $(+)$ on $C_{K}$. Note that the equation of motion for $G^{\lessgtr}(1,2)$ does not contain the source term $\delta(1-2)$ since the time $\tau_{1}$ and $\tau_{2}$ are on different branches, and in the non-interacting case, one has $\left[i \partial_{\tau_{1}}-\bar{h}_{0}(1)\right] g^{\lessgtr}(1,2)=0$, and $\left[-i \partial_{\tau_{2}}-\bar{h}_{0}(2)\right] g^{\lessgtr}(1,2)=0$.

Using the relationships between the different GFs given in Appendix Appendix B, one can calculate the integral equations for the different Green's functions [2, 9, 10, 11, 12, 13, 14, and one finds that the retarded and advanced $G^{r, a}$ Green's functions obey a Dyson-like equation:

$$
G^{r}(1,2)=g^{r}(1,2)+\int \mathrm{d}(34) g^{r}(1,3) \Sigma^{r}(3,4) G^{r}(4,2),
$$

(likewise for $G^{a}$ ) where the time integrations are on the real times $\int_{-\infty}^{+\infty} \mathrm{d} t_{i}$.

The lesser and greater $G^{\lessgtr}$ GFs obey the following quantum kinetic equation:

$$
G^{\lessgtr}(1,2)=\int \mathrm{d} 3 \mathrm{~d} 4\left(1+G^{r} \Sigma^{r}\right) g^{\lessgtr}\left(1+\Sigma^{a} G^{a}\right)+G^{r} \Sigma^{\lessgtr} G^{a},
$$

given in a compact notation. Eq.(66) is valid within the conventional assumption of the Keldysh time-loop contour formalism where the interactions are switched on

adiabatically. The most general solution for the lesser/greater Green's function can be found in Appendix A of Ref. [17].

\subsection{The time-ordered Green's function on $C_{K}$}

Following the rules of decomposition of the time-loop contour $C_{K}$ onto real-time given in Appendix Appendix A, one can expand the equation for the time-ordered GF $G^{t}=G^{++}$ 
as follows (using compact notation)

$$
\begin{aligned}
G^{++} & =g^{++}+(g \Sigma G)^{++} \\
& =g^{++}+g^{++} \Sigma^{++} G^{++} \\
& -g^{++} \Sigma^{<} G^{>}-g^{<} \Sigma^{>} G^{++}+g^{<\Sigma^{--}} G^{++}
\end{aligned}
$$

hence we get

$$
\begin{aligned}
G^{t}= & g^{t}\left(1+\Sigma^{t} G^{t}-\Sigma^{<} G^{>}\right) \\
& -g^{<}\left(\Sigma^{\tilde{t}} G^{>}-\Sigma^{>} G^{t}\right) .
\end{aligned}
$$

After further manipulation, using Appendix A and Appendix B, we can show that the second line in Eq. (86) becomes $g^{<}\left(\Sigma^{\tilde{t}} G^{>}-\Sigma^{>} G^{t}\right)=g^{<}(\Sigma G)^{>}$. Hence the full equation for the time-ordered GF is

$$
G^{t}=g^{t}\left(1+\Sigma^{t} G^{t}-\Sigma^{<} G^{>}\right)+g^{<}(\Sigma G)^{>} .
$$

From the equation of motion of GF on the Keldysh contour, we find

$$
g^{<}(\Sigma G)^{>} \equiv \int \mathrm{d} 2 g^{<}(1,2)\left[\mathrm{i} \partial_{t_{2}}-\bar{h}_{0}(2)\right] G^{>}(2,3)
$$

and by using the (adjoint) equation of motion of the non-interacting GF $g^{<}$, i.e. $\left[-\mathrm{i} \partial_{t_{2}}-\bar{h}_{0}(2)\right] g^{>}(1,2)=0$, we can integrate Eq. (10) by part and find that

$$
\left[g^{<}(\Sigma G)^{>}\right](1,3) \equiv \int \mathrm{d} x_{2} \mathrm{i}\left[g^{<}\left(1, x_{2} t\right) G^{>}\left(x_{2} t, 3\right)\right]_{t=+\infty}-\mathrm{i}\left[g^{<}\left(1, x_{2} t\right) G^{>}\left(x_{2} t, 3\right)\right]_{t=-\infty},
$$

which represents a boundary condition term for the time variable (after all we are solving a first order differential equation in time).

Hence Eq. (8) is transformed into

$$
\begin{aligned}
G^{t}(1,2) & =g^{t}(1,2)+\int \mathrm{d}(34) g^{t}(1,3)\left(\Sigma^{t}(3,4) G^{t}(4,2)-\Sigma^{<}(3,4) G^{>}(4,2)\right) \\
& -\int \mathrm{d} x_{2} \mathrm{i}\left[g^{<}\left(1, x_{2} t\right) G^{>}\left(x_{2} t, 3\right)\right]_{t=+\infty}-\mathrm{i}\left[g^{<}\left(1, x_{2} t\right) G^{>}\left(x_{2} t, 3\right)\right]_{t=-\infty},
\end{aligned}
$$

or equivalently (in compact notation)

$$
G^{t}=g^{t}\left(1+\Sigma^{t} G^{t}-\Sigma^{<} G^{>}\right)+\text {boundary condition. }
$$

Eq. (12), equivalently Eq. (8)), is the most general result for the time-ordered Green's function and is valid for the non-equilibrium case at finite and zero temperature. It also clearly shows that the time-ordered Green's function $G^{t}(1,2)$ does not follow a Dysonlike equation. However this is not a closed equation, in contradistinction with the Dyson equations for the advanced and retarded components, since it also involves greater and lesser components for the GF and the self-energy. In the general non-equilibrium 
conditions, one rather works with the closed equations obtained for the retarded and advanced GF which are related to spectroscopic properties, and with the quantum kinetic equations obtained for the lesser and greater GF. Though at equilibrium, the lesser and greater components of GF or a self-energy are related to the respective spectral functions and the equilibrium distribution. Hence we recover closed equations for the time-ordered GF.

We also establish in Section 4 the formal links with the approach of Hedin and Lundqvist and with the Matsubara temperature GF formalism.

It would be interesting to find some conditions under which the the boundary condition term in Eq. (13) vanishes. At equilibrium, because of the principle of adiabatic switching (on/off) of the interactions in the remote past/future, the initial state returns (up to a phase factor) to itself in the far future. Therefore it would be tempting to consider that the the two terms defining the boundary condition are the same at $t= \pm \infty$ and that the boundary condition term vanishes. However this is not that case, as shown in Section 4 .

At non-equilibrium, the problem is different and the solution may depend on the size of the system. For an infinite system (a large system in the thermodynamic limit), in some cases, we would like to use the so-called memory-loss theorem [17, 18, 19]: the long time limit of the lesser (or greater) components of the GF or of the self-energy vanish. Hence in that case, the boundary condition term may vanish (though this needs to be proven). This corresponds to cases when the system "thermalise with the environment". For finite size systems at non-equilibrium, no "thermalisation" is possible and the boundary condition term play an important role in the solution of dynamical equation of the time-ordered GF.

Before turning onto the equilibrium condition, let us make two further general remarks. One could note that from the definition $G^{r}(1,2)=G^{t}(1,2)-G^{<}(1,2)$ (see Appendix Appendix B) or equivalently $G^{t}(1,2)=G^{r}(1,2)+G^{<}(1,2)$, one could have gueseds that $G^{t}$ does not obey a Dyson equation since $G^{r}$ does, but not $G^{<}$ [20]. Futhermore we have also checked that we can also recover, as expected, our result Eq. (13) by using the Kadanoff-Baym formalism which is somewhat different but equivalent to the Keldysh time-loop approach [21].

\subsection{At equilibrium}

We now turn onto the special case of equilibrium for which the Green's functions techniques based on many-body perturbation theory were orginally derived.

At equilibriumn, as in the steady state, all quantities $X\left(t, t^{\prime}\right)$ (GFs and self-energies) depend only on the time difference $X\left(t-t^{\prime}\right)$. One can then Fourier transform the quantity $X\left(t-t^{\prime}\right)$ and work in the real-frequency representation $X(\omega)$.

The main result of the paper, Eq. (12), is recast as:

$$
G^{t}(\omega)=g^{t}(\omega)\left(1+\Sigma^{t}(\omega) G^{t}(\omega)-\Sigma^{<}(\omega) G^{>}(\omega)\right)-g^{<}(\omega)(\Sigma(\omega) G(\omega))^{>},
$$


in compact notation (i.e. omitting the explicit integrals over space).

It should be noticed that the last term $\Sigma^{<} G^{>}$in Eq. (12) satisfies the detailed balance equation at equilibrium [10]: $\Sigma^{<}(\omega) G^{>}(\omega)=\Sigma^{>}(\omega) G^{<}(\omega)$.

Eq. (12) can also be recast as

$$
\left[\left[g^{t}(\omega)\right]^{-1}-\Sigma^{t}(\omega)\right] G^{t}(\omega)+\Sigma^{<}(\omega) G^{>}(\omega)=1,
$$

ignoring the boundary conditions term.

Furthermore, at equilibrium, the lesser and greater components of either a GF or a self-energy $\left(X^{\lessgtr}\right)$ can be expressed in terms of the corresponding advanced and retarded quantity and a distribution function [3, 4, 22, 5, 23, 7, 24, 25, 26, i.e.

$$
X^{\lessgtr}(\omega)=-f^{\lessgtr, \mathrm{eq}}(\omega)\left(X^{r}(\omega)-X^{a}(\omega)\right),
$$

and spectral function $A^{X}=\left(X^{r}(\omega)-X^{a}(\omega)\right) /(-2 \pi i)$.

At equilibrium and for a system of fermions, $f^{<, \text {eq }}$ is given by the Fermi-Dirac distribution function $f^{\mathrm{eq}}(\omega)=\left[1+\exp \beta\left(\omega-\mu^{\mathrm{eq}}\right)\right]^{-1}$ and $f^{>\text {,eq }}=f^{\mathrm{eq}}-1$ (with $\beta=1 / k T$ ). Eq. (16) follows from the Kubo-Martin-Schwinger relationship between the lesser and greater component at equilibrium: $X^{>, \mathrm{eq}}(\omega)=-\mathrm{e}^{\beta\left(\omega-\mu^{\mathrm{eq}}\right)} X^{<, \mathrm{eq}}(\omega)$.

Hence we can easily see that

$$
\Sigma^{<}(\omega) G^{>}(\omega)=(2 \pi)^{2} f^{\mathrm{eq}}(\omega)\left(1-f^{\mathrm{eq}}(\omega)\right) A^{\Sigma}(\omega) A^{G}(\omega)
$$

Therefore in the limit of zero temperature, there is no contribution from $\Sigma^{<} G^{>}$ since $f^{\mathrm{eq}}\left(1-f^{\mathrm{eq}}\right)=0$ (this property holds for any product $X^{<} Y^{>}$, and hence for the boundary condition term $\left.g^{<}(\Sigma G)^{>}\right)$, and the time-ordered GF $G^{t}$ obeys a conventional Dyson-like equation $G^{t}(\omega)=g^{t}(\omega)\left[1+\Sigma^{t}(\omega) G^{t}(\omega)\right]$.

It is only in the case of equilibrium at zero temperature that $G^{t}$ follows a Dyson equation otherwise at finite temperature $G^{t}$ obeys a modified Dyson equation, Eq. (15), including the extra term $\Sigma^{<} G^{>}$and the boundary condition term $g^{<}(\Sigma G)^{>}$.

In the following section, we are going to show how Hedin and Lundqvist noticed the necessity to introduce correction terms in the real-time Green's function at finite temperature [16. They found it necessary to introduce extra terms in the Dyson equation of the real-time Green's function at finite temperature to recover the expected

behaviour of the independent particle case. The terms introduced ad hoc by Hedin and Lundqvist correspond exactly to the $\Sigma^{<} G^{>}$term that we have obtained in a natural way from the use of the time-loop contour.

\section{Real-time Green functions at finite temperature}

In this section, we retrace the analysis made by Hedin and Lundqvist (section 17 of Ref. [16]) on the temperature Green's functions. The definition for the real-time GF at finite temperature follows from the zero temperature case by using an ensemble average 
for the time-ordered product of fermion operators instead of a ground state expectation value, i.e.

$$
G(1,2)=-\mathrm{i} \operatorname{Tr}\left[\rho_{G} \mathcal{T} \Psi(1) \Psi^{\dagger}(2)\right],
$$

where the statistical operator (density matrix) $\rho_{G}=Z_{G}^{-1} \exp -\beta\left(H-\mu^{\mathrm{eq}} N\right)$ and the partition function $Z_{G}=\operatorname{Tr}\left[\rho_{G}\right]$ are given for the grand canonical ensemble.

After Fourier transform, we can write the time-ordered GF using a spectral representation just as in the zero temperature case [3, 4, 16]:

$$
G(\omega)=\int \mathrm{d} \omega^{\prime} A\left(\omega^{\prime}\right)\left[\frac{1-f^{\mathrm{eq}}\left(\omega^{\prime}\right)}{\omega-\omega^{\prime}+\mathrm{i} \eta}+\frac{f^{\mathrm{eq}}\left(\omega^{\prime}\right)}{\omega-\omega^{\prime}-\mathrm{i} \eta}\right],
$$

where we omit the spatial variables.

From Eq. (19), we can obtain the real and imaginary parts of $G(\omega)$ :

$$
\Re e G(\omega)=P V \int \mathrm{d} \omega^{\prime} \frac{A\left(\omega^{\prime}\right)}{\omega-\omega^{\prime}},
$$

and

$$
\Im m G(\omega)=\pi\left[2 f^{\mathrm{eq}}(\omega)-1\right] A(\omega) .
$$

Hedin and Lundqvist noticed that by considering the conventional Dyson equation for the time-ordered GF: $[\omega-\epsilon-\Sigma(\omega)] G(\omega)=1$, one derives the following spectral function

$$
A(\omega)=\frac{B(\omega)}{[\omega-\epsilon-\Re e \Sigma(\omega)]^{2}+\pi^{2} B(\omega)^{2}\left[2 f^{\mathrm{eq}}-1\right]^{2}},
$$

with the spectral representation for the self-energy $\Im m \Sigma(\omega)=\pi\left[2 f^{\mathrm{eq}}(\omega)-1\right] B(\omega)$.

However Eq. (22) does not have the proper limit in $\delta(\omega-\epsilon)$ for the independent particle case where $\Re e \Sigma(\omega)=0$ and $B(\omega) \rightarrow 0$.

To palliate this discrepancy, Hedin and Lundqvist introduced ad hoc a correction term in the conventional Dyson equation that $G$ and $\Sigma$ must obey. Their modified Dyson equation reads

$$
[\omega-\epsilon-\Sigma(\omega)] G(\omega)+(2 \pi)^{2} f^{\mathrm{eq}}\left(1-f^{\mathrm{eq}}\right) A(\omega) B(\omega)=1 .
$$

From this modified Dyson equation, one can calculate the imaginary part of $G(\omega)$ and obtain the spectral function $A(\omega)$

$$
A(\omega)=\frac{B(\omega)}{[\omega-\epsilon-\Re e \Sigma(\omega)]^{2}+\pi^{2} B(\omega)^{2}},
$$

as for the zero temperature limit, it has the proper limit $A(\omega) \rightarrow \delta(\omega-\epsilon)$ for the limit $\Re e \Sigma(\omega)=0$ and $B(\omega) \rightarrow 0$.

Hedin and Lundqvist conclude "that the rather messy definition of $\Sigma$ in Eq. (23) shows that it is better if, at finite temperature, we can work with concepts other that the straight-forward generalisation in Eq. (18) of the zero temperature Green's function. 
One possibility is to use the Matsubara technique with temperature Green's functions, or the close related Martin and Schwinger technique ..." [16].

It is interesting to note that the "messy" definition Eq. (23) is actually the correct result for the time-ordered GF $G^{t}$ at finite temperature. Eq. (23) is strictly equivalent to Eq. (15) when one notices that in the definition of $\Sigma^{<} G^{>}$in Eq. (17) one deals with the same spectral functions, i.e. $A \equiv A^{G}$ and $B \equiv A^{\Sigma}$.

So by using the Keldysh time-loop contour, we have found in a natural way, almost straightforward, the proper modified Dyson equation for $G^{t}$ at equilibrium and this, without the need of introducing correction terms with no proper justification, other than the need to recover the correct behaviour for $A^{G}(\omega)$ in the non-interacting case.

To conclude this section, one could rephrase our result by saying that the timeordered GF $G^{t}$ at equilibrium and finite temperature obeys a Dyson equation $G^{t}=$ $g^{t}+g^{t} \bar{\Sigma} G^{t}$ only if one defines the corresponding self-energy $\bar{\Sigma}$ as $\bar{\Sigma}=\Sigma^{t}-\Sigma^{<} G^{>}\left(G^{t}\right)^{-1}$.

\section{Recovering $G^{t}$ from the equilibrium formalism and imaginary-time temperature Green's functions}

In the next section, we show that the proper modified Dyson equation for $G^{t}$ can be obtained from the knowledge of the spectral function $A^{X}(\omega)=\left(X^{r}(\omega)-X^{a}(\omega)\right) /-\mathrm{i} 2 \pi$, with $X$ being a GF or a self-energy. We also discuss the connection of our result with the Matsubara temperature Green's functions.

We extract the spectral function $A^{G}$ using the Dyson equations for $G^{r, a}$ :

$$
G^{r}-G^{a}=g^{r}-g^{a}+g^{r} \Sigma^{r} G^{r}-g^{a} \Sigma^{a} G^{a} .
$$

Using the definitions of the spectral functions $A^{G}$ and $A^{\Sigma}$ and the fact that $\Re e G^{r}=\Re e G^{a}=$ P.V. $\int A^{G}\left(\omega^{\prime}\right) /\left(\omega-\omega^{\prime}\right)=\Re e G^{t}$ as in Eq. (20) (with similar relations for the self-energy $\Sigma$ ), we find

$$
\begin{aligned}
A^{G}=A^{g} & +A^{g}\left(\Re e \Sigma . \Re e G-\pi^{2} A^{\Sigma} A^{G}\right) \\
& +\Re e(g) .\left(\Re e \Sigma . A^{G}+A^{\Sigma} . \Re e G\right) .
\end{aligned}
$$

The time-ordered GF $G^{t}(\omega)$ can then be obtained from its spectral representation Eq. (19) using the expression of the spectral function $A^{G}(\omega)$ given by Eq. (26). The first term in the RHS of Eq. (26) generates the non-interacting time-ordered GF $g^{t}(\omega)$, while the two other terms regrouped in $D(\omega)=A^{g}\left(\Re e \Sigma . \Re e G-\pi^{2} A^{\Sigma} A^{G}\right)+$ $\Re e(g) .\left(\Re e \Sigma . A^{G}+\Re e G . A^{\Sigma}\right)$ give a real part P.V. $\int D\left(\omega^{\prime}\right) /\left(\omega-\omega^{\prime}\right)$ and an imaginary part $\pi\left[2 f^{\mathrm{eq}}(\omega)-1\right] D(\omega)$. The proper result for the modified Dyson equation of $G^{t}(\omega)$ can be obtained after lengthy algebraic derivations. Instead we now show another just as valid shorter route to end up with the correct result.

We consider the different contributions of a conventional Dyson $g^{t}+g^{t} \Sigma^{t} G^{t}$. From the spectral representation, we have already generated the term in $g^{t}$ from $A^{g}$, so we are left with the $g^{t} \Sigma^{t} G^{t}$. 
We first concentrate on the product $\Sigma^{t} G^{t}$ :

$$
\begin{aligned}
& \Sigma^{t} G^{t} \\
& =\left(\Re e \Sigma+\mathrm{i} \pi\left(2 f^{\mathrm{eq}}-1\right) A^{\Sigma}\right)\left(\Re e G+\mathrm{i} \pi\left(2 f^{\mathrm{eq}}-1\right) A^{G}\right) \\
& =\left(\Re e \Sigma . \Re e G-\pi^{2} A^{\Sigma} A^{G}-(2 \pi)^{2} f^{\mathrm{eq}}\left(f^{\mathrm{eq}}-1\right) A^{\Sigma} A^{G}\right) \\
& \quad \quad+\mathrm{i} \pi\left(2 f^{\mathrm{eq}}-1\right)\left(\Re e \Sigma \cdot A^{G}+A^{\Sigma} . \Re e G\right) \\
& =\left(\Re e \Sigma . \Re e G-\pi^{2} A^{\Sigma} A^{G}+\Sigma^{<} G^{>}\right) \\
& \quad+\mathrm{i} \pi\left(2 f^{\mathrm{eq}}-1\right)\left(\Re e \Sigma \cdot A^{G}+A^{\Sigma} . \Re e G\right),
\end{aligned}
$$

where we have introduced, in the last equality, the definition of the product $\Sigma^{<} G^{>}$given in Eq. (17). We can see that the correction term in $\Sigma^{<} G^{>}$appears already at this level.

Hence one can see that

$$
\begin{aligned}
& 1+\Sigma^{t} G^{t}-\Sigma^{<} G^{>} \\
& =\left(1+\Re e \Sigma \Re e G-\pi^{2} A^{\Sigma} A^{G}\right)+\mathrm{i}\left(2 f^{\mathrm{eq}}-1\right) \pi\left(\Re e \Sigma \cdot A^{G}+A^{\Sigma} \Re e G\right) .
\end{aligned}
$$

To finish our derivation, we first consider the imaginary part of $g^{t}\left(1+\Sigma^{t} G^{t}-\Sigma^{<} G^{>}\right)$. By using the definition for $g^{t}$ from Eqs. (20) and (21) $g^{t}=\Re e(g)+i\left(2 f^{\mathrm{eq}}-1\right) \pi A^{g}$, we obtain:

$$
\begin{aligned}
& \Im m\left[g^{t}\left(1+\Sigma^{t} G^{t}-\Sigma^{<} G^{>}\right)\right] \\
& =\Im m\left(g^{t}\right) \Re e\left(1+\Sigma^{t} G^{t}-\Sigma^{<} G^{>}\right)+\Re e\left(g^{t}\right) \Im m\left(1+\Sigma^{t} G^{t}-\Sigma^{<} G^{>}\right) \\
& =\left(2 f^{\mathrm{eq}}-1\right) \pi A^{g}\left(1+\Re e \Sigma \Re e G-\pi^{2} A^{\Sigma} A^{G}\right)+\Re e(g)\left(2 f^{\mathrm{eq}}-1\right) \pi\left(\Re e \Sigma \cdot A^{G}+A^{\Sigma} \Re e G\right) \\
& =\left(2 f^{\mathrm{eq}}-1\right) \pi A^{G} \\
& =\Im m G^{t} .
\end{aligned}
$$

For the real part of $g^{t}\left(1+\Sigma^{t} G^{t}-\Sigma^{<} G^{>}\right)$, we find that

$$
\begin{aligned}
& \Re e\left[g^{t}\left(1+\Sigma^{t} G^{t}-\Sigma^{<} G^{>}\right)\right] \\
& =\Re e\left(g^{t}\right) \Re e\left(1+\Sigma^{t} G^{t}-\Sigma^{<} G^{>}\right)-\Im m\left(g^{t}\right) \Im m\left(1+\Sigma^{t} G^{t}-\Sigma^{<} G^{>}\right) \\
& =\Re e(g)\left(1+\Re e \Sigma \Re e G-\pi^{2} A^{\Sigma} A^{G}\right)-\left(2 f^{\mathrm{eq}}-1\right) \pi A^{g}\left(2 f^{\mathrm{eq}}-1\right) \pi\left(\Re e \Sigma \cdot A^{G}+A^{\Sigma} \Re e G\right) \\
& =\Re e(g)\left(1+\Re e \Sigma \Re e G-\pi^{2} A^{\Sigma} A^{G}\right)-\pi^{2} A^{g}\left(\Re e \Sigma \cdot A^{G}+A^{\Sigma} \Re e G\right) \\
& -4 f^{\mathrm{eq}}\left(f^{\mathrm{eq}}-1\right) \pi A^{g} \pi\left(\Re e \Sigma \cdot A^{G}+A^{\Sigma} \Re e G\right) .
\end{aligned}
$$

We can use the properties of the Hilbert transform [27, 28, 29], i.e. $\mathcal{H}[\mathcal{H}[f]]=-f$ and the Bedrosian indentity $\mathcal{H}[f g]]=f \mathcal{H}[g]=g \mathcal{H}[f]$, to show that:

$$
\begin{aligned}
& \Re e G^{t}=\mathcal{H}\left[\pi A^{G}\right] \\
& =\mathcal{H}\left[\pi A^{g}\left(1+\Re e \Sigma \Re e G-\pi^{2} A^{\Sigma} A^{G}\right)\right]+\mathcal{H}\left[\pi \Re e(g)\left(\Re e \Sigma . A^{G}+A^{\Sigma} \Re e G\right)\right] \\
& \left.=\mathcal{H}\left[\pi A^{g}\right]\left(1+\Re e \Sigma \Re e G-\pi^{2} A^{\Sigma} A^{G}\right)+\mathcal{H}[\pi \Re e(g)]\left(\Re e \Sigma \cdot A^{G}+A^{\Sigma} \Re e G\right)\right] \\
& \left.=\Re e(g)\left(1+\Re e \Sigma \Re e G-\pi^{2} A^{\Sigma} A^{G}\right)-\pi^{2} A^{g}\left(\Re e \Sigma . A^{G}+A^{\Sigma} \Re e G\right)\right],
\end{aligned}
$$


since $\Re e(g)=\mathcal{H}\left[\pi A^{g}\right]$, and $\mathcal{H}[\pi \Re e(g)]=\pi^{2} \mathcal{H}\left[\mathcal{H}\left[A^{g}\right]\right]$.

Hence Eq. (31) becomes

$$
\Re e G^{t}=\Re e\left[g^{t}\left(1+\Sigma^{t} G^{t}-\Sigma^{<} G^{>}\right)\right]+4 f^{\mathrm{eq}}\left(f^{\mathrm{eq}}-1\right) \pi A^{g} \pi\left(\Re e \Sigma \cdot A^{G}+A^{\Sigma} \Re e G\right),
$$

When considering that, by definition at equilibrium, we have:

$$
\begin{aligned}
& g^{<}=-f^{\mathrm{eq}}\left(g^{r}-g^{a}\right)=\mathrm{i} f^{\mathrm{eq}} 2 \pi A^{g} \\
& (\Sigma G)^{>}=-\left(f^{\mathrm{eq}}-1\right)\left((\Sigma G)^{r}-(\Sigma G)^{a}\right),
\end{aligned}
$$

and $(\Sigma G)^{r}-(\Sigma G)^{a}=\Sigma^{r} G^{r}-\Sigma^{a} G^{a}=-2 \mathrm{i}\left(\pi A^{\Sigma} \Re e G+\Re e \Sigma . \pi A^{G}\right)$, we can see that, as expect, the last term in Eq. (32) is just equal to $g^{<(\Sigma G)^{>}}$.

Hence we recover once more the modified Dyson equation for the time-ordered GF:

$$
G^{t}(\omega)=g^{t}(\omega)\left(1+\Sigma^{t}(\omega) G^{t}(\omega)-\Sigma^{<}(\omega) G^{>}(\omega)\right)-g^{<}(\omega)(\Sigma(\omega) G(\omega))^{>}
$$

in a indirect way from the spectral functions.

We now briefly comment on the imaginary-time temperature Green's functions. The definition of the temperature GF is, in form, similar to a time-ordered Green's function:

$$
\mathcal{G}\left(\mathbf{x} \tau, \mathbf{x}^{\prime} \tau^{\prime}\right)=-\operatorname{Tr}\left[\rho_{G} \mathcal{T}_{\tau} \Psi(\mathbf{x} \tau) \Psi^{\dagger}\left(\mathbf{x}^{\prime} \tau^{\prime}\right)\right]
$$

where one uses imaginary times $\tau=$ it instead of real time $t$, and the $\mathcal{T}_{\tau}$ orders the operators according to their value of $\tau$, with the smallest at the right [3, 4].

The temperature Green's function $\mathcal{G}(1,2)$ follows a conventional Dyson-like equation:

$$
\mathcal{G}(1,2)=\mathcal{G}_{0}(1,2)+\int \mathrm{d}(34) \mathcal{G}_{0}(1,3) \Sigma(3,4) \mathcal{G}(4,2)
$$

where the time integrations run over $\tau_{i}$ from 0 to $\beta$ (and $\mathcal{G}_{0}$ is the temperature Green's function for the non-interacting system).

At equilibrium, the quantities depend only on the "time" difference $\tau_{1}-\tau_{2}$ and $\mathcal{G}\left(\mathbf{x}, \mathbf{x}^{\prime}, \tau-\tau^{\prime}\right)$ is periodic over the range $2 \beta$ (or periodic (antiperiodic) over the range $[0, \beta]$ for boson (fermion)). Therefore $\mathcal{G}$ can be expanded in a Fourier series with coefficients $\mathcal{G}\left(\mathbf{x}, \mathbf{x}^{\prime}, \omega_{n}\right)$ given in terms of the Matsubara frequencies $\omega_{n}$ being integer even (odd) numbers of $\pi / \beta$ for boson (fermion). Each Fourier component obeys also a Dyson equation:

$$
\mathcal{G}\left(\omega_{n}\right)=\mathcal{G}_{0}\left(\omega_{n}\right)+\mathcal{G}_{0}\left(\omega_{n}\right) \Sigma\left(\omega_{n}\right) \mathcal{G}\left(\omega_{n}\right)
$$

(in compact notation).

There is a well defined mathematical connection between the temperature GF $\mathcal{G}\left(\omega_{n}\right)$ and the real-time GF, this is obtained from the analytical continuation from the complex frequency $i \omega_{n}$ on the real frequency $\omega$ (and changing, when needed, the sum over the Matsubara frequencies onto integrals $\left.1 / \beta \sum_{\omega_{n}} \ldots \rightarrow \int \mathrm{d} \omega / 2 \pi \ldots\right)$.

However, even if $\mathcal{G}$ is an imaginary-time-ordered $\mathrm{GF}$, it does not transform into the real-time-ordered GF. The analytical continuation $\mathrm{i} \omega_{n} \rightarrow \omega+\mathrm{i} \eta$ transforms the 
temperature GF into the retarded real-time GF (in energy representation) [3, 4] : $\mathcal{G}\left(\omega_{n}\right) \rightarrow G^{r}(\omega)$. Similarly we have $\mathcal{G}\left(\omega_{n}\right) \rightarrow G^{a}(\omega)$ for $\mathrm{i} \omega_{n} \rightarrow \omega-\mathrm{i} \eta$. This is also consistent with the fact the $G^{r, a}$ obeys a Dyson equation, as much as $\mathcal{G}$ does too.

In order to get the time-ordered GF $G^{t}(\omega)$ for the temperature GF $\mathcal{G}\left(\omega_{n}\right)$, one has to adopt the following procedure [4]: from $\mathcal{G}\left(\omega_{n}\right)$ and the analytical continuation, one obtains $G^{r}(\omega)$ and $G^{a}(\omega)$ from which one extracts the spectral function $A^{G}(\omega)$ which is then used to built the time-ordered $\mathrm{GF}^{t}(\omega)$ from the spectral representation Eq. (19). Following this procedure, we have shown above that one can obtain the modified Dyson equation for $G^{t}(\omega)$.

As another example, we provide in Appendix C similar calculations for a specific system, i.e. a finite size system at finite temperature, described by the Hubbard Hamiltonian.

\section{Conclusion}

We have derived the dynamical equation for the time-ordered GF $G^{t}\left(t, t^{\prime}\right)$ in the most general case of non-equilibrium and finite temperature. In the equilibrium case, we have shown that the corresponding time-ordered GF $G^{t}(\omega)$ obeys a Dyson equation only in the limit of zero temperature. At finite temperature, the Dyson equation for $G^{t}(\omega)$ needs to be modified by a correction term. We have shown that the correction term correspond exactly to the corrections introduced $a d$ hoc by Hedin and Lundqvist in their treatment of the real-time GF at finite temperature. We have also shown that these corrections can be obtained from the Matsubara temperature GF following the appropriate protocol for the analytical continuation from the imaginary frequency onto the real frequency and the spectral representation of the time-ordered GF. Table 1 summarizes the different GFs formalism and the corresponding dynamical equations for the different GFs.

Our work leads to two important conclusions. First, the Keldysh time-loop contour formalism to the GFs, though developed to deal with non-equilibrium conditions, is also extremely appropriate to the study of the equilibrium properties of the system. It leads, in a more natural and straightforward way, to the correct dynamical equations of the GFs. Second, any formalisms based on the resolution of the Dyson equation for the time-ordered GF, as those used for example in electronic structure and excitations calculations, are valid only at equilibrium and in the zero temperature limit. These formalisms include GF calculations ranging from the $G W$ approximation [16, 30, 31] to more recent work on self-consistent GF equations involving four-point propagators [32]. Beyond the zero temperature, correction terms in the Dyson equation for $G^{t}(\omega)$ must be taken into account, or one needs to work with the Matsubara formalism for $\mathcal{G}\left(i \omega_{n}\right)$. Such temperature effects were also found for the $T$-matrix approximation in a real-time formalism in Ref. [33. 


\begin{tabular}{|c|c|c|c|}
\hline & $\begin{array}{l}\text { Non-equilibrium } \\
\text { all } T\end{array}$ & $\begin{array}{l}T \neq 0 \\
\text { at equilibrium }\end{array}$ & $\begin{array}{l}T=0 \\
\text { at equilibrium }\end{array}$ \\
\hline$G\left(\tau, \tau^{\prime}\right)$ & $\begin{array}{l}\text { Dyson eqn on } C_{K}: \\
G=g+g \Sigma G\end{array}$ & & \\
\hline $\begin{array}{l}G^{r}, G^{a} \\
\text { in }\left(t, t^{\prime}\right) \text { or } \\
\quad(\omega)\end{array}$ & $\begin{array}{l}\text { Dyson eqn: } \\
G^{r, a}=g^{r, a}+g^{r, a} \sum^{r, a} G^{r, a}\end{array}$ & $\begin{array}{l}\text { Dyson eqn: } \\
G^{r, a}=g^{r, a}+g^{r, a} \Sigma^{r, a} G^{r, a}\end{array}$ & $\begin{array}{l}\text { Dyson eqn: } \\
G^{r, a}= \\
g^{r, a}+g^{r, a} \Sigma^{r, a} G^{r, a}\end{array}$ \\
\hline $\begin{array}{c}G^{\lessgtr} \\
\text { in }\left(t, t^{\prime}\right) \text { or } \\
(\omega)\end{array}$ & $\begin{array}{l}\text { QKE: } \\
G^{\lessgtr}=G^{r} \Sigma^{\lessgtr} G^{a} \\
+\left(1+G^{r} \Sigma^{r}\right) g^{\lessgtr}\left(1+\Sigma^{a} G^{a}\right)\end{array}$ & $\begin{array}{l}\text { QKE: } \\
G^{\lessgtr}(\omega)= \\
-f^{\lessgtr, \mathrm{eq}}(\omega)\left(G^{r}-G^{a}\right)(\omega)\end{array}$ & $\begin{array}{l}\text { QKE: } \\
G^{\lessgtr}= \\
-f^{\lessgtr, \mathrm{eq}}\left(G^{r}-G^{a}\right)\end{array}$ \\
\hline $\begin{array}{c}G^{t} \\
\text { in }\left(t, t^{\prime}\right) \text { or } \\
(\omega)\end{array}$ & $\begin{array}{l}\text { Modified Dyson eqn: } \\
G^{t}=g^{t}\left(1+\Sigma^{t} G^{t}-\Sigma^{<} G^{>}\right) \\
+ \text {boundary condition }\end{array}$ & $\begin{array}{l}\text { Modified Dyson eqn: } \\
G^{t}=g^{t}\left(1+\Sigma^{t} G^{t}-\Sigma^{<} G^{>}\right) \\
+g^{<}(\Sigma G)^{>}\end{array}$ & $\begin{array}{l}\text { Dyson eqn: } \\
G^{t}=g^{t}+g^{t} \Sigma^{t} G^{t}\end{array}$ \\
\hline 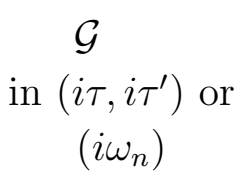 & & $\begin{array}{l}\text { Dyson eqn: } \\
\mathcal{G}=\mathcal{G}_{0}+\mathcal{G}_{0} \Sigma \mathcal{G}\end{array}$ & \\
\hline
\end{tabular}

Table 1. Summary of the different Green's function formalisms in different situations (equilibrium/non-equilibrium, finite/zero temperature). The rows show the relevant Green's functions and the different dynamical equations (Dyson, modified Dyson, or Quantum kinetic equation QKE) for each situation. Compact notation is used, involving integration of space and time (unless the energy $\omega$ representation is used) coordinates.

\section{Acknowledgments}

We thank Martin Stankovksi for pointing us to the early work of Hedin and Lundqvist on real-time GF at finite-temperature; Falk Tandetzky, Sangeeta Sharma and Pina Romaniello for careful reading of the paper and valuable comments; Arno Schindlmayr for useful comments on the Matsubara formalism; and Rex Godby for discussions. 


\section{Appendix A. Rules for continuation on time-loop contour $C_{K}$}

For the following products $P_{(i)}\left(\tau, \tau^{\prime}\right)$ on the time-loop contour $C_{K}$,

$$
\begin{aligned}
P_{(2)}\left(\tau, \tau^{\prime}\right) & =\int_{C_{K}} \mathrm{~d} \bar{\tau} A(\tau, \bar{\tau}) B\left(\bar{\tau}, \tau^{\prime}\right) \\
P_{(3)} & =\int_{C_{K}} A B C \\
P_{(n)} & =\int_{C_{K}} A_{1} A_{2} \ldots A_{n},
\end{aligned}
$$

we have the following rules for the different components $P_{(i)}^{x}\left(t, t^{\prime}\right)$ on the real-time axis: $(x=r, a,>,<)$

$$
\begin{aligned}
P_{(2)}^{\gtrless}\left(t, t^{\prime}\right) & =\int \mathrm{d} \bar{t} A^{r}(t, \bar{t}) B^{\gtrless}\left(\bar{t}, t^{\prime}\right)+A^{\gtrless}(t, \bar{t}) B^{a}\left(\bar{t}, t^{\prime}\right) \\
P_{(3)}^{\gtrless} & =\int_{t} A^{\gtrless} B^{a} C^{a}+A^{r} B^{\gtrless} C^{a}+A^{r} B^{r} C^{\gtrless} \\
P_{(n)}^{r} & =\int_{t} A_{1}^{r} A_{2}^{r} \ldots A_{n}^{r}, \quad P_{(n)}^{a}=\int_{t} A_{1}^{a} A_{2}^{a} \ldots A_{n}^{a} .
\end{aligned}
$$

\section{Appendix B. Relationship between the different Green's functions and self-energies}

The relations between the different components of the Green's functions and selfenergies on the Keldysh time-loop contour are given as usual, with $X^{\eta_{1} \eta_{2}}(12) \equiv$ $G^{\eta_{1} \eta_{2}}(12), \Sigma^{\eta_{1} \eta_{2}}(12)$ or $g^{\eta_{1} \eta_{2}}(12)$.

$$
\begin{aligned}
X^{r}=X^{t}-X^{<} & =X^{>}-X^{\tilde{t}} \\
X^{a}=X^{t}-X^{>} & =X^{<}-X^{\tilde{t}} \\
X^{t}+X^{\tilde{t}} & =X^{<}+X^{>} \\
X^{>}-X^{<} & =X^{r}-X^{a}
\end{aligned}
$$

The usual lesser and greater projections are defined respectively as $X^{<} \equiv X^{+-}$and $X^{>} \equiv X^{-+}$, and the usual time-ordered (anti-time-ordered) as $X^{t}=X^{++}\left(X^{\tilde{t}}=X^{--}\right)$.

\section{Appendix C. Example for a finite system at equilibrium}

In this section, we consider in finite system, with interaction, for which analytical expressions are derivable for the different GFs and we check the validity of Eq. (14) for such a system at equilibrium.

We work with the single-site Hubbard Hamiltonian given by:

$$
H=\sum_{\sigma=\uparrow, \downarrow} \varepsilon_{0 \sigma} n_{\sigma}+U n_{\uparrow} n_{\downarrow}
$$


where $n_{\sigma}$ is the occupation number operator of the spin $\sigma$. In the absence of magnetic field, the system is spin degenerate when $\varepsilon_{0 \sigma}=\varepsilon_{0}$. We consider this case in the following. The system has 4 different states upon the level occupation $n_{\sigma}=0, n_{\uparrow}=1$ and $n_{\downarrow}=0$, $n_{\uparrow}=0$ and $n_{\downarrow}=1, n_{\uparrow}=1$ and $n_{\downarrow}=1$ with respective energy $0, \varepsilon_{0}, \varepsilon_{0}, 2 \varepsilon_{0}+U$. One can calculate the advanced and retarded GFs at equilibrium (and finite temperature, for the finite size system "connected" to a thermal bath at temperature $T$ ) following the usual prescription [3, 4]. Within the Lehmann representation, we find

$$
G^{r, a}(\omega)=\frac{1}{Z}\left\{\frac{1+\mathrm{e}^{-\beta \varepsilon_{0}}}{\omega-\varepsilon_{0} \pm \mathrm{i} \eta}+\frac{\mathrm{e}^{-\beta \varepsilon_{0}}+\mathrm{e}^{-\beta\left(2 \varepsilon_{0}+U\right)}}{\omega-\varepsilon_{0}-U \pm \mathrm{i} \eta}\right\},
$$

with the partition function $Z=1+2 \mathrm{e}^{-\beta \varepsilon_{0}}+\mathrm{e}^{-\beta\left(2 \varepsilon_{0}+U\right)}, \beta=1 / k T$ and $\eta \rightarrow 0^{+}$.

We define the thermal occupancy $f$ as follows

$$
f=\frac{\mathrm{e}^{-\beta \varepsilon_{0}}+\mathrm{e}^{-\beta\left(2 \varepsilon_{0}+U\right)}}{Z} \text { and } 1-f=\frac{1+\mathrm{e}^{-\beta \varepsilon_{0}}}{Z} .
$$

In the non-interacting case $(U=0)$, one recovers the usual GF expressions for a single electronic level, $G^{r, a}=1 /\left(\omega-\varepsilon_{0} \pm \mathrm{i} \eta\right)$ and $f=f^{\mathrm{eq}}=1 /\left(1+\mathrm{e}^{\beta \varepsilon_{0}}\right)$ the Fermi distribution (with the chemical potential $\mu^{\mathrm{eq}}=0$ taken as the reference of energy for all the expression given in this Appendix).

Eq. (C.2) can be rewritten as

$$
G^{r, a}(\omega)=\frac{1-f}{\omega-\varepsilon_{0} \pm \mathrm{i} \eta}+\frac{f}{\omega-\varepsilon_{0}-U \pm \mathrm{i} \eta},
$$

with the usual relationships between the imaginary and real of $G^{r, a}(\omega)$ based on the properties of the Hilbert transform $\mathcal{H}[\pi \delta(\omega-E)]=\mathcal{P} \mathcal{V} \frac{1}{(\omega-E)}($ with $\mathcal{P} \mathcal{V}$ standing for the Cauchy principal value).

We now define the self-energy $\Sigma^{r, a}(\omega)$ corresponding to $G^{r, a}(\omega)$ as follows:

$$
\frac{1-f}{\omega-\varepsilon_{0} \pm \mathrm{i} \eta}+\frac{f}{\omega-\varepsilon_{0}-U \pm \mathrm{i} \eta}=\frac{1}{\omega-\varepsilon_{0}-\Sigma^{r, a}(\omega)} .
$$

One can separate the real and imaginary parts of both sides of the equality, using $\Sigma^{r / a}=\Re e \Sigma \pm \mathrm{i} \Im m \Sigma$ :

$$
\begin{array}{r}
(1-f) \mathcal{P} \mathcal{V} \frac{1}{\omega-\varepsilon_{0}}+f \mathcal{P} \mathcal{V} \frac{1}{\omega-\varepsilon_{0}-U} \mp \mathrm{i} \pi\left[(1-f) \delta\left(\omega-\varepsilon_{0}\right)+f \delta\left(\omega-\varepsilon_{0}-U\right)\right] \\
=\frac{\omega-\varepsilon_{0}-\Re e \Sigma \mp \mathrm{i} \Im m \Sigma}{\left(\omega-\varepsilon_{0}-\Re e \Sigma\right)^{2}+(\Im m \Sigma)^{2}} .
\end{array}
$$

In the LHS of Eq. (C.6), the real and imaginary parts are related to each other via an Hilbert transform as explained above. Hence this must held as well for the RHS of Eq. (C.6). Indeed, from Eq. (C.6), we have

$$
\omega-\varepsilon_{0}-\Re e \Sigma=\left[(1-f) \mathcal{P} \mathcal{V} \frac{1}{\omega-\varepsilon_{0}}+f \mathcal{P} \mathcal{V} \frac{1}{\omega-\varepsilon_{0}-U}\right] \times \mathcal{N}(\omega),
$$


and

$$
\Im m \Sigma=\left[(1-f) \delta\left(\omega-\varepsilon_{0}\right)+f \delta\left(\omega-\varepsilon_{0}-U\right)\right] \times \mathcal{N}(\omega),
$$

with $\mathcal{N}^{-1}(\omega)=\left(\omega-\varepsilon_{0}-\Re e \Sigma\right)^{2}+(\Im m \Sigma)^{2}$. Combining Eqs. (C.7) and (C.8), we find that

$\mathcal{N}=$

$$
\left(\left[(1-f) \mathcal{P} \mathcal{V} \frac{1}{\omega-\varepsilon_{0}}+f \mathcal{P} \mathcal{V} \frac{1}{\omega-\varepsilon_{0}-U}\right]^{2}+\left[(1-f) \delta\left(\omega-\varepsilon_{0}\right)+f \delta\left(\omega-\varepsilon_{0}-U\right)\right]^{2}\right) \mathcal{N}^{2},
$$

hence another definition for $\mathcal{N}(\omega)$.

By using three properties of the Hilbert transform [27, 28, 29], $\mathcal{H}[\mathcal{H}[g(u)]]=-g(u)$, and the Bedrosian identity for the Hilbert transform of product functions $\mathcal{H}[g(u) h(u)]=$ $g(u) \mathcal{H}[h(u)]=h(u) \mathcal{H}[g(u)]$, one can show that from Eqs.(C.7) and (C.8), we have:

$$
\begin{aligned}
\mathcal{H}\left[\left(\omega-\varepsilon_{0}-\Re e \Sigma\right) \mathcal{N}\right] & =-\mathcal{H}[\Re e \Sigma]=\left((1-f) \mathcal{H}\left[\mathcal{P} \mathcal{V} \frac{1}{\omega-\varepsilon_{0}}\right]+f \mathcal{H}\left[\mathcal{P} \mathcal{V} \frac{1}{\omega-\varepsilon_{0}-U}\right]\right) \mathcal{N} \\
& =-\Im m \Sigma,
\end{aligned}
$$

hence $\Re e \Sigma(\omega)=-\mathcal{H}[\Im m \Sigma(\omega)]$ as expected, see for example Eq. (20). The first equality comes from the fact that $\mathcal{H}\left[\left(\omega-\varepsilon_{0}\right) \mathcal{N}\right]=0$ when $\mathcal{N}^{-1}\left(\omega=\varepsilon_{0}\right) \neq 0$.

Now that we have established the usual relationships between the imaginary and real parts of both the advanced and retarded GFs and self-energies $(X)$ for our finite size model, i.e. $X^{r, a}(\omega)=\Re e X(\omega) \mp \mathrm{i} \Im m X(\omega)$ and $X^{r}(\omega)-X^{a}(\omega)=-2 \pi \mathrm{i} A^{X}$ (with $\left.A^{X}(\omega)= \pm \frac{1}{\pi} \Im m X^{r / a}(\omega)\right)$, and $\Re e X(\omega)=\mathcal{P} \mathcal{V} \int \mathrm{d} \omega^{\prime} \frac{A^{X}\left(\omega^{\prime}\right)}{\omega-\omega^{\prime}}=-\mathcal{H}[\Im m X(\omega)]$, we can go further along and define as usual the lesser and greater components (at equilibrium): $X^{<}(\omega)=-f\left(X^{r}(\omega)-X^{a}(\omega)\right)=2 \pi \mathrm{i} f A^{X}(\omega)$, and $X^{>}(\omega)=-(f-1)\left(X^{r}(\omega)-X^{a}(\omega)\right)=$ $2 \pi \mathrm{i}(f-1) A^{X}(\omega)$, as well as the time-ordered components: $\Re e X^{t}(\omega)=\Re e X^{r, a}(\omega)$ and $\Im m X^{t}(\omega)=\pi(2 f-1) A^{X}(\omega)$. We recall that for our finite size system at equilibrium, the distribution $f$ is given by Eq. (C.3).

We can now check the validity of Eq. (14) for our model system. For that we follow the prescriptions given in Section 4. We first evaluate the imaginary part of $g^{t}+g^{t} \Sigma^{t} G^{t}-g^{t} \Sigma^{<} G^{>}$:

$$
\begin{aligned}
& \Im m\left[g^{t}+g^{t} \Sigma^{t} G^{t}-g^{t} \Sigma^{<} G^{>}\right]= \\
& \Im m g^{t}\left(1+\Re e \Sigma^{t} \Re e G^{t}-\Im m \Sigma^{t} \Im m G^{t}-\Sigma^{<} G^{>}\right)+\Re e g^{t}\left(\Im m \Sigma^{t} \Re e G^{t}+\Re e \Sigma^{t} \Im m G^{t}\right),
\end{aligned}
$$

since $\Sigma^{<} G^{>}$is real. Using the different definitions, we find

$$
\begin{aligned}
& \Im m\left[g^{t}+g^{t} \Sigma^{t} G^{t}-g^{t} \Sigma^{<} G^{>}\right]= \\
& \Im m g^{t}\left(1+\Re e \Sigma \Re e G-\pi^{2}(2 f-1)^{2} A^{\Sigma} A^{G}-(2 \pi)^{2} f(1-f) A^{\Sigma} A^{G}\right) \\
& +\Re e g^{t}\left(\pi(2 f-1) A^{\Sigma} \Re e G+\Re e \Sigma \pi(2 f-1) A^{G}\right) .
\end{aligned}
$$


Using the definition $\Im m g^{t}=\pi(2 f-1) A^{g}$, we end up with

$$
\begin{aligned}
& \Im m\left[g^{t}+g^{t} \Sigma^{t} G^{t}-g^{t} \Sigma^{<} G^{>}\right]= \\
& \pi(2 f-1)\left[A^{g}\left(1+\Re e \Sigma \Re e G-\pi^{2} A^{\Sigma} A^{G}\right)+\Re e g\left(A^{\Sigma} \Re e G+\Re e \Sigma A^{G}\right)\right],
\end{aligned}
$$

where the term in brakets is just the definition of the spectral function $A^{G}$ given by Eq. (26). Hence we find that

$$
\Im m\left[g^{t}+g^{t} \Sigma^{t} G^{t}-g^{t} \Sigma^{<} G^{>}\right]=\pi(2 f-1) A^{G}=\Im m G^{t}
$$

as expected. One can proceed along the same lines to establish the relation for the real parts, using the different properties of the Hilbert transform mentioned above. Therefore, as expected, Eq. (14) is valid for the finite size system, at equilibrium, described by Hamiltonian Eq. (C.1).

\section{References}

[1] Pines D 1961 The Many-Body Problem (New York: W.A. Benjamin)

[2] Kadanoff L P and Baym G 1962 Quantum Statistical Mechanics (New York: W.A. Benjamin)

[3] Abrikosov A A, Gorkov L P and Dzyaloshinski I E 1963 Methods of Quantum Field Theory in Statistical Physics (New York: Dover)

[4] Fetter A L and Walecka J D 1971 Quantum Theory of Many-Particle Systems (New York: McGrawHill)

[5] Rammer J and Smith H 1986 Review of Modern Physics 58323

[6] Mahan G D 1990 Many-Particle Physics (New York: Plenum Press)

[7] Bruus H and Flensberg K 2004 Many-body Quantum Theory in Condensed Matter (Oxford: Oxford University Press)

[8] Dickhoff W H and Neck D V 2008 Many-body theory exposed! (Singapore: World Scientific)

[9] Keldysh L 1965 Sov. Phys. JETP 201018

[10] Danielewicz P 1984 Annals of Physics 152239

[11] chao Chou K, bin Su Z, lin Hao B and Yu L 1985 Physics Reports 1181

[12] Wagner M 1991 Physical Review B 446104

[13] van Leeuwen R, Dahlen N E, Stefanucci G, Almbladh C O and von Barth U 2006 Lecture Notes in Physics 70633

[14] Rammer J 2007 Quantum Field Theory of Non-Equilibrium States (Cambridge: Cambridge University Press)

[15] Schwinger J 1961 J. Math. Phys. 2407

[16] Hedin L and Lundqvist S 1969 Effects of Electron-Electron and Electron-Phonon Interactions on the One-Electron States of Solids vol 23 (New York: Academic Press)

[17] Stefanucci G and Almbladh C O 2004 Phys. Rev. B 69195318

[18] Doyon B and Andrei N 2006 Phys. Rev. B 73245326

[19] Stefanucci G 2007 Phys. Rev. B 75195115

[20] We have also found that when calculating the retarded GF $G^{r}=G^{t}-G^{<}$from Eq. (12) for $G^{t}$ and Eq. (6) for $G^{<}$, we recover the conventional Dyson equation for $G^{r}$ but only thanks to the presence of the term $\Sigma^{<} G^{>}$in the definition of $G^{t}$.

[21] One can check that Eq. (8-27a) and Eq. (8-27b) in Ref. 2] are equivalent to the equation of motion Eq. (2) for $G^{\lessgtr}$, i.e. $L(1) G^{\lessgtr}\left(1,1^{\prime}\right)=(\Sigma G)^{\lessgtr}\left(1,1^{\prime}\right)$ with the one-point operator $L(1)=i \partial_{t_{1}}-\bar{h}_{0}(1)$ and the relation $(X Y)^{\lessgtr}=X^{r} Y^{\lessgtr}+X \lessgtr Y^{a}$. Then by using the definition of the time-ordered GF $G^{t}(1,2)=-\mathrm{i}\left\langle\mathcal{T} \Psi(1) \Psi^{\dagger}(2)\right\rangle$ given in terms of $G^{\lessgtr}: G^{t}(1,2)=\theta\left(t_{1}-t_{2}\right) G^{>}(1,2)+\theta\left(t_{2}-\right.$ 
$\left.t_{1}\right) G^{<}(1,2)$ and calculating directly its equation of motion $L(1) G^{t}(1,2)$, one can recover the expression of the modified Dyson equation Eq. (13).

[22] Lipavský P, Špička V and Velický B 1986 Physical Review B $\mathbf{3 4} 6933$

[23] Meden V, Wöhler C, Fricke J and Schönhammer K 1995 Physical Review B 525624

[24] Vasko F T and Raichev O E 2005 Quantum Kinetic Theory and Applications: Electrons, Photons, Phonons (New York: Springer Science+Business Media Inc)

[25] Ness H, Dash L and Godby R W 2010 Physical Review B 82085426

[26] Kita T 2010 Progress of Theoretical Physics 123581

[27] Bedrosian E, 1963 Proc. IEEE 51868

[28] Xu Y and Yan D 2006 Proc. Amer. Math. Soc. 1342719

[29] Poularikas A. D. 1999 The Handbook of Formulas and Tables for Signal Processing (Boca Raton: CRC Press LLC). Chapter 15 on "The Hilbert Transform".

[30] Onida G, Reining L and Rubio A 2002 Rev. Mod. Phys. 74 601-659

[31] Ness H, Dash L K, Stankovski M and Godby R W 2011 Physical Review B 84195114

[32] Starke R and Kresse G 2012 Physical Review B 85075119

[33] Canivell V, Garrido L, Miguel M S and Seglar P 1978 Physical Review A 17480 